\title{
Modeling and Optimization in Fibrous Materials
}

\author{
Abhijit Majumdar $^{1} \cdot$ Yordan Kyosev $^{2}$
}

Published online: 13 June 2015

(C) The Institution of Engineers (India) 2015

Fibrous materials are increasingly being used in various technical applications including healthcare, protection, sports, industrial and composites. For ages, fibrous materials have been developed based on intuition, thumb rules and trial and error approach. However, in recent years, development of models to understand the manufacturing process and structure-property relationship of fibrous materials has emerged as a challenging area of research. Tensile, impact, bending, compression, shear, heat transfer, moisture-vapour transfer, fluid permeability and UV transmission are some of the properties of fibrous materials which have been modelled extensively by mathematical, analytical, statistical and intelligent techniques. The physical and performance requirements of fibrous materials are dependent on the end use. Sometimes, these requirements are conflicting with each other and thus optimization becomes essential. These models are often used to optimize

This special issue is a modest endeavour to compile some modelling and optimization related research work in the domain of fibrous materials. We hope that this issue will kindle some interest among the textile researchers to take this area forward.

Abhijit Majumdar

majumdar@textile.iitd.ac.in

Yordan Kyosev

yordan.kyosev@hs-niederrhein.de

1 Department of Textile Technology, Indian Institute of Technology, Delhi, India

2 Faculty of Textile and Clothing Technology, Hochschule Niederrhein - University of Applied Sciences, Mönchengladbach, Germany the properties of the final product by varying the materials properties, structural and process parameters.

This special issue entitled "Modeling and Optimization in Fibrous Materials" contains 11 papers. These papers encompass the applications of various modeling techniques in fibres, yarns, fabrics and carpets.

The first paper is a thematic one which deals with various types of mathematical models and their principles. The second paper presents an insight into the mechanics of $1 \times 1$ rib loop formation system on dial and cylinder machine. The degree of agreement between predicted and measured values of loop length and cam force justifies the efficacy of the model. The third paper proposes a theoretical model for predicting warp yarn tension during weaving based on the dynamic nature of shed geometry. The study also reveals that the developed yarn tension peak values are different for the extreme positions of a heald.

The fourth paper deals with a strain sensitive textile based elastomeric tape sensor development. The weave structure, number of conductive threads and rubber thread tension has been optimized by using the Box-Behnken design of experiment method. The fifth paper describes the optimization of the aqua splicer parameters namely opening time, splicing time, splice length and duration of water joining for lycra-cotton core spun yarn using Taguchi experimental design.

The sixth paper presents Eyring's non-linear viscoelastic model to simulate stress-strain behaviour of polyester and viscose filaments. The complex mathematical equations of Eyring's model for curve fitting are handled by genetic algorithm. The authors demonstrate that that Eyring's model can be used to simulate the stress-strain behaviours of polyester and viscose filaments with reasonable accuracy. The seventh paper proposes different 
possibilities of micromagnetic modeling of magnetic fibers or coatings. It presents an overview of calculation times for different dimensions of magnetic materials, indicating the limits due to available computer performance.

The eighth to eleventh papers deal with various soft computing methods like Artificial Neural Network (ANN), Adaptive Network based Fuzzy Inference System (ANFIS) and genetic algorithm for textile modeling and optimization. The eighth paper presents an endeavor to predict the percentage yarn strength utilization in cotton woven fabrics using ANN approach. The results indicate that while an increase in the number of load bearing or transverse yarns increases the percentage yarn strength utilization, an increase in the float length and the crimp percentage in the yarns have a detrimental effect. The ninth paper propounds a new approach of fabric engineering using ANN and genetic algorithm. The three ANN models has been developed for the prediction of drape coefficient, air permeability and thermal resistance. The fabric engineering problem has been solved using genetic algorithm. The tenth paper presents prediction of bending rigidity of cotton woven fabrics by ANN and two hybrid soft computing methodologies, namely neuro-genetic modeling (GANN) and ANFIS. The GANN model, in particular, shows better prediction accuracy than the other two models. The last paper presents the application of ANN modeling for the prediction of abrasion resistance of Persian handmade wool carpets.
Abhijit Majumdar obtained Bachelors Degree from Calcutta University with first class first position in Textile Technology program. Subsequently, he acquired M.Tech. degree in Textile Engineering from IIT Delhi and Ph.D. in Production Engineering from Jadavpur University, Kolkata. He also holds M.B.A. degree from IIT Delhi with specialization in Operations Management. He has worked in industries like Voltas Limited and Vardhman Group. He joined IIT Delhi as Assistant Professor in 2007. Currently he is associate professor in textile engineering group. His research areas include protective textiles, soft computing applications and operations management. He has completed three research projects, as Principal Investigator, funded by DST and CSIR, India. He has published 50 research papers in International refereed Journals. He is the Associate Editor of Journal of the Institution of Engineers (India) Series E (Chemical and Textile Engineering).

Yordan Kyosev obtained his Masters in Technique and Technology of Textile and Clothing and Applied Mathematics from Technical University of Sofia, Bulgaria. He completed his Ph.D. in Textile Engineering from the same University. He worked as Assistant Professor until 2005 in Technical University of Sofia, Bulgaria. He received Alexander von Humboldt Fellowship for Postdoctoral study at the Institute of Textile Technology (ITA) [Institut fürTextiltechnik] of the RWTH Aachen University, Germany. Prof. Kyosev joined as Professor in the Textile and Clothing Technology, HochschuleNiederrhein - University of Applied Sciences, Germany in the year 2006. He has published numerous Journal papers, Conference presentations, several Book Chapters about Modeling of Textiles. He has also authored a book on Fuzzy Logic with Prof. K. Peeva. Prof. Kyosev has published a Monograph titled, "Braiding Technology for Textiles", which becomes the main textbook in this area. He has also developed industrial software, which is used in modelling and simulation of textile structures for several companies. 